OPEN ACCESS

Edited by:

Volker Rasche,

University of UIm, Germany

Reviewed by:

Zhifen Liu,

First Hospital of Shanxi Medical

University, China

Zhenghua Hou,

Southeast University, China

${ }^{*}$ Correspondence:

Wenbin Guo

guowenbin76@csu.edu.cn

Shuguang Luo

luoshuguang@stu.gxmu.edu.cn

Specialty section

This article was submitted to Applied Neuroimaging,

a section of the journal

Frontiers in Neurology

Received: 10 July 2021 Accepted: 04 October 2021 Published: 05 November 2021

Citation:

Wei S, Chen X, Xiao Y, Jiang W, Yin Q

Lu C, Yang L, Wei J, Liu Y, Li W.

Tang J, Guo W and Luo S (2021) Abnormal Network Homogeneity in the Right Superior Medial Frontal

Gyrus in Cervical Dystonia.

Front. Neurol. 12:729068

doi: 10.3389/fneur.2021.729068

\title{
Abnormal Network Homogeneity in the Right Superior Medial Frontal Gyrus in Cervical Dystonia
}

\begin{abstract}
Shubao Wei ${ }^{1}$, Xiuqiong Chen ${ }^{1}$, Yousheng Xiao ${ }^{2}$, Wenyan Jiang ${ }^{2}$, Qiong Yin ${ }^{2}$, Chunhui Lu ${ }^{2}$, Lu Yang ${ }^{2}$, Jing Wei ${ }^{2}$, Yang Liu ${ }^{2}$, Wenmei $\mathrm{Li}^{3}$, Jingqun Tang ${ }^{2}$, Wenbin Guo ${ }^{4 *}$ and Shuguang Luo ${ }^{2 *}$

${ }^{1}$ Department of Rehabilitation Medicine, Jiangbin Hospital of Guangxi Zhuang Autonomous Region, Nanning, China, ${ }^{2}$ Department of Neurology, The First Affiliated Hospital of Guangxi Medical University, Nanning, China, ${ }^{3}$ Department of Radiology, The First Affiliated Hospital of Guangxi Medical University, Nanning, China, ${ }^{4}$ National Clinical Research Center for Mental Disorders, Department of Psychiatry, The Second Xiangya Hospital of Central South University, Changsha, China
\end{abstract}

Background: Increasing evidence from modern neuroimaging has confirmed that cervical dystonia (CD) is caused by network abnormalities. Specific brain networks are known to be crucial in patients suffering from CD. However, changes in network homogeneity $(\mathrm{NH})$ in $\mathrm{CD}$ patients have not been characterized. Therefore, the purpose of this study was to investigate the $\mathrm{NH}$ of patients with $\mathrm{CD}$.

Methods: An automated $\mathrm{NH}$ method was used to analyze resting-state functional magnetic resonance (fMRI) data from 19 patients with $\mathrm{CD}$ and 21 gender- and age-matched healthy controls $(\mathrm{HC})$. Correlation analysis were conducted between $\mathrm{NH}$, illness duration and symptom severity measured by the Tsui scale.

Results: Compared with the $\mathrm{HC}$ group, $\mathrm{CD}$ patients showed a lower $\mathrm{NH}$ in the right superior medial frontal gyrus. No significant correlations were found between abnormal $\mathrm{NH}$ values and illness duration or symptom severity.

Conclusion: Our findings suggest the existence of abnormal $\mathrm{NH}$ in the default mode network (DMN) of CD patients, and thereby highlight the importance of the DMN in the pathophysiology of CD.

Keywords: cervical dystonia, resting-state functional magnetic resonance, network homogeneity, default mode network, superior medial frontal gyrus

\section{INTRODUCTION}

Cervical dystonia (CD) is a disorder of the nervous system characterized by involuntary sustained contractions of the cervical musculature, causing an abnormal and involuntary rotation or tilt of the head in specific directions (1). The head may typically turn resulting in torticollis, laterocollis, anterocollis, or retrocollis (2). CD is the most common type of focal dystonia, and the first symptoms are frequently exhibited between 40 and 60 years of age. The disorder is often accompanied by head tremors and chronic neck pain (3). Abnormal posture can cause pain and lead to significant disability in daily living activities, such as reading and driving $(4,5)$, which can lead to unemployment $(6,7)$. Indeed, there has been reported a substantial negative influence of $\mathrm{CD}$ on employment, with CD-related pain as a particularly important factor (8). Moreover, CD patients sustain significant psychosocial disability and a decline in their quality of life. Therefore, 
it is important to diagnose patients with $\mathrm{CD}$ and offer these patients an effective therapy, however, the pathological mechanism of CD is still poorly understood.

It is widely accepted that deficient motor control in dystonia is related to basal ganglia dysfunction, ultimately resulting in a systems-level loss of inhibitory function (9-12). Recently, emerging evidence suggests the probability of widespread abnormalities in brain networks in which the basal ganglia are important nodes. This introduced the hypothesis that CD can be attributed to the dysfunction of specific brain networks, although their precise role in CD is currently uncertain.

Through the developments of neuroimaging techniques, it is now possible to investigate details of structural changes and neuronal activity in the brain that are potentially involved in the pathophysiology of CD. For example, several structural neuroimaging studies have reported micro-structural changes in specific brain regions of CD patients, including the basal ganglia, thalamus, cerebellum, motor cortex, and supplementary motor cortices (13-21). Functional magnetic resonance imaging (fMRI) studies provide the most consistent results $(22,23)$. Furthermore, in task-free fMRI research, CD patients were shown to have altered functional brain connectivity in a couple of restingstate networks as compared with those of healthy controls (22). Thus, cervical dystonia is suggested to be a disorder caused by the dysfunction of multiple neural networks. Recently, we have studied regional homogeneity ( $\mathrm{ReHo}$ ) changes in $\mathrm{CD}$, and confirmed that abnormal ReHo existed in brain regions of the "pain matrix", salience network (the right insula and bilateral middle cingulate gyrus), motor network (the right precentral gyrus), cerebellum and medial prefrontal cortex (MPFC) (24). However, it remains unclear if the network homogeneity $(\mathrm{NH})$ is affected in CD patients.

Over recent years, a methods to analyze resting-state fMRI data have provided a new way to study the previously neglected field of intrinsic network organization (25). The method named $\mathrm{NH}$, which is an unbiased assessment of the homogeneity of a neural network, was first used to study the default mode network (DMN) of attention-deficit/hyperactivity disorder by Uddin et al. (25). NH is a voxel-wise that examines the correlation of a voxel with all other voxels belonging to a specific network of interest. The NH existed its advantages that have been detail stated in our previous studies $(26,27)$. Homogeneity is defined as the average correlation of the time series of any given voxel with the time series of all other voxels within the network (25). So far, NH has been investigated in studies of individuals with somatization disorder (26), major depressive disorder (28), schizophrenia (27) and their unaffected counterparts (29).

In this study, we used the $\mathrm{NH}$ method to study patients with CD. Previously, we have used Global-brain functional connectivity (GFC), voxel-mirrored homotopic connectivity (VMHC) and ReHo to analyze fMRI data of patients with CD. Results showed that the GFC and the VMHC in the supplementary motor area (SMA) were significantly decreased $(30,31)$. Furthermore, a negative correlation was found between the VMHC values in the SMA with the severity of dystonia (31). Additionally, CD patients showed increased ReHo in the right cerebellum crus I and decreased ReHo in the right superior
MPFC. The right precentral gyrus, right insula, and bilateral middle cingulated gyrus also showed increased ReHo values. A significantly positive correlation was observed between ReHo in the right cerebellum crus I and symptom severity (24). Therefore, $\mathrm{CD}$ patients had alterations in brain networks of the "pain matrix", salience network, motor network and DMN. Given the $\mathrm{NH}$ method was restricted to reveal the neuro-activities of DMN, thus we expected the NH method making new contributions by exploring $\mathrm{NH}$ in the $\mathrm{DMN}$ to understanding the pathological mechanism of CD. Based on previous findings, we hypothesize that $\mathrm{CD}$ patients would show abnormal $\mathrm{NH}$, particularly the $\mathrm{DMN}$, and that may further positively correlated with clinical data such as illness duration and symptom severity.

\section{MATERIALS AND METHODS}

\section{Subjects}

This study, which was approved by the ethics committee of the First Affiliated Hospital, Guangxi Medical University, China, was performed in an outpatient setting in the Department of Neurology of The First Affiliated Hospital. Patients were diagnosed based on the 2011 EFNS (European Federation of Neurological Societies) guideline on the diagnosis and treatment of primary dystonias. Patients who matched the following criteria were included in the study: (1) idiopathic cervical dystonia; (2) no history of botulinum toxin treatment, related drug therapy, or operation in the previous 3 months; (3) no history of serious physical or neuropsychiatric disorders; and (4) right-handedness.

Healthy controls (HC) were simultaneously recruited from the community. They were group-matched in gender, age, and were all right-handed. The exclusion criteria for HC individuals were as follows: (1) diagnosis of secondary spasmodic torticollis; (2) any history of severe physical or neuropsychiatric disorders; and (3) any family history of psychiatric or neurological diseases in their first-degree relatives. Only subjects who had no contraindications for MRI or exhibited no abnormalities in conventional MRI scans were included.

The Tsui scale (32) was used to measure the symptom severity of CD of all patients. Every subject was given information relating to study procedures and provided written informed consent.

\section{Image Acquisition}

Resting-state image acquisition was performed on a Siemens 3.0 T scanner (Erlangen, Germany). All subjects underwent scans with foam padding and earplugs to minimize head movement and reduce scanner noise. Every participant was asked to lie still and relax, eyes closed, and remain awake but avoid thinking of anything in particular. Subsequently, we confirmed that each subject had not fallen asleep during the session. For each subject, the following parameters were used for echo-planar imaging sequence acquisition: repetition time/echo time $(\mathrm{TR} / \mathrm{TE})=$ $2,000 / 30 \mathrm{~ms}$, slice thickness $=4 \mathrm{~mm}$, flip angle $=90^{\circ}$, gap $=$ $0.4 \mathrm{~mm}$, matrix $=64 \times 64, \mathrm{FOV}=24 \times 24 \mathrm{~cm}$, number of volumes $=250$. 


\section{Data Pre-processing}

Data pre-processing was performed using Matlab (33) (Mathworks) data processing assistant for resting-state fMRI (DPARSF) (34). The primary process included: slice timing, realignment, spatial normalization, registration to the MNI template, and temporal bandpass filtering. Details of preprocessing are presented in our previous study (26). We excluded the participants who had more than $2^{\circ}$ of angular rotation in each axis and $2 \mathrm{~mm}$ of translation in the $\mathrm{x}^{-}, \mathrm{y}^{-}$, or z-direction.

\section{NH Analysis}

After data pre-processing, we performed $\mathrm{NH}$ analysis on Matlab (33) (Mathworks). The equation to calculate the $\mathrm{NH}$ of $\mathrm{CD}$ patients and HCs has been previously described by Uddin et al. (25). Briefly, the correlation coefficients of each voxel were computed against all other voxels within the DMN mask and averaged to generate the NH maps. Subsequently, the NH maps were smoothed by a Gaussian kernel of $8 \mathrm{~mm}$ with full-width at half-maximum and used in further analysis.

\section{Statistical Analysis}

Demographic and clinical information, such as sex and age were recorded. The Chi-square test was used to compare qualitative variables, whereas the two-sample $t$-test was used to compare quantitative variables $(p<0.05)$. The $\mathrm{NH}$ analysis were completed using REST with a two-sample $t$-test. Multiple comparisons were conducted using a Gaussian random field (GRF) correction (voxel significance: $p<0.001$, cluster significance: $p<0.005$ ), and the level of significance was set at the corrected $p<0.05$.

Moreover, the mean $\mathrm{NH}$ values of brain regions that were defined as regions of interest (ROIs) that showed abnormal $\mathrm{NH}$ were extracted. Subsequently, further correlation analysis between these $\mathrm{NH}$ values and illness duration and Tsui scores in the patient group $(p<0.05)$ were performed. Correlation analysis of mean $\mathrm{NH}$ values of clusters and patient age were performed using Pearson's correlations, and correlation analysis of illness duration and Tsui total score were performed using Spearman's correlations.

\section{Reproducibility}

Given the small sample size, we used split-half and leave-oneout validations to assess the reproducibility of the present results. First, we randomly selected $10 \mathrm{CD}$ patients and 11 healthy controls from the two groups and analyzed using the same statistical tests as those employed for the total number of patients. In parallel, group comparisons may significantly lose statistical power in the split-half analysis. Thus, we also performed leaveone-out validations of the reproducibility and robustness of these results without reduced statistical power. Specifically, we excluded one CD patient from the group and performed the same group comparisons based on the permutated sample (e.g., $18 \mathrm{CD}$ vs. 21 healthy controls). Consequently, a total of 19 two-sample $t$-test images were generated. Based on these images, numerous tests for each voxel revealed significant group differences across
TABLE 1 | Demographics and clinical characteristics of the patients and the controls.

\begin{tabular}{lccc}
\hline $\begin{array}{l}\text { Variables } \\
\text { (mean } \pm \text { standard deviation) }\end{array}$ & Patients & Controls & p-value \\
\hline Gender (female/male) & $10 / 9$ & $15 / 6$ & $0.220^{\mathrm{a}}$ \\
Age, years & $38.74 \pm 10.71$ & $39.62 \pm 6.62$ & $0.759^{\mathrm{b}}$ \\
Illness duration, months & $24.29 \pm 31.26$ & & \\
Tsui & $16.32 \pm 4.45$ & & \\
\hline
\end{tabular}

Tsui, Tsui scale.

a The $p$-value for gender distribution in the two groups was obtained by chi-square test. ${ }^{b}$ The $p$-values were obtained by two sample t-tests.

TABLE 2 | Difference in head motion of the patients and the controls.

\begin{tabular}{lrrrrrrr}
\hline Testing & \multicolumn{3}{c}{ Translation } & & \multicolumn{3}{c}{ Rotation } \\
\cline { 2 - 4 } \cline { 5 - 7 } & \multicolumn{1}{c}{$\mathbf{x}$} & $\mathbf{y}$ & $\mathbf{z}$ & & $\mathbf{x}$ & $\mathbf{y}$ & $\mathbf{z}$ \\
\hline$T$ & -0.943 & -0.781 & -0.033 & -0.378 & -0.845 & -1.148 \\
$P$ & 0.352 & 0.439 & 0.974 & & 0.707 & 0.403 & 0.258 \\
\hline
\end{tabular}

The $p$ values were obtained by two sample t-tests. $x, y, z$, coordinates of the MNI space. $x$, $y, z$ represented of translation in the $x-, y$-, or $z$-direction and angular rotation in each axis.

all 19 two-sample $t$-tests; these differences were calculated as the reproducibility of $\mathrm{NH}$ between $\mathrm{CD}$ patients and HCs.

\section{RESULTS}

\section{Subjects}

There were no subject excluded due to any contraindications for MRI or shown changes under conventional MRI scans. No participants were excluded due to falling asleep during image acquisition. Two patients were excluded due to excessive head movement. Therefore, 19 patients and $21 \mathrm{HCs}$ were included in this study. As listed in Table 1, there were no significant differences between the patient and control groups in gender ratio ( $t$-tests, $p=0.220$ ) or age (Chi-square test, $p=0.759$ ). There were no difference in head motion of the residual patients and HCs. The detail results were listed in Table 2. Additionally, in the CD group, 12 patients were bilaterally affected, while the remaining patients were affected on the left side only. A total of 17 patients complained of painful neck muscles, and 18 of 19 CD patients reported sensory tricks. Sensory tricks are known as an abnormal posture and involuntary movements of the head and neck that can be temporarily improved by specific behaviors, including light touching the lower part of the cheek, jaw and posterior neck, leaning against the wall, carrying a backpack or keeping something in the mouth.

\section{NH: Between-Group Comparison}

Compared with HCs, CD patients showed a lower NH in the right superior medial frontal gyrus. None of the brain regions showed a significantly increased $\mathrm{NH}$ in CD patients compared with those of HCs. Group comparisons of NH values are shown in (Figure 1; Table 3). 


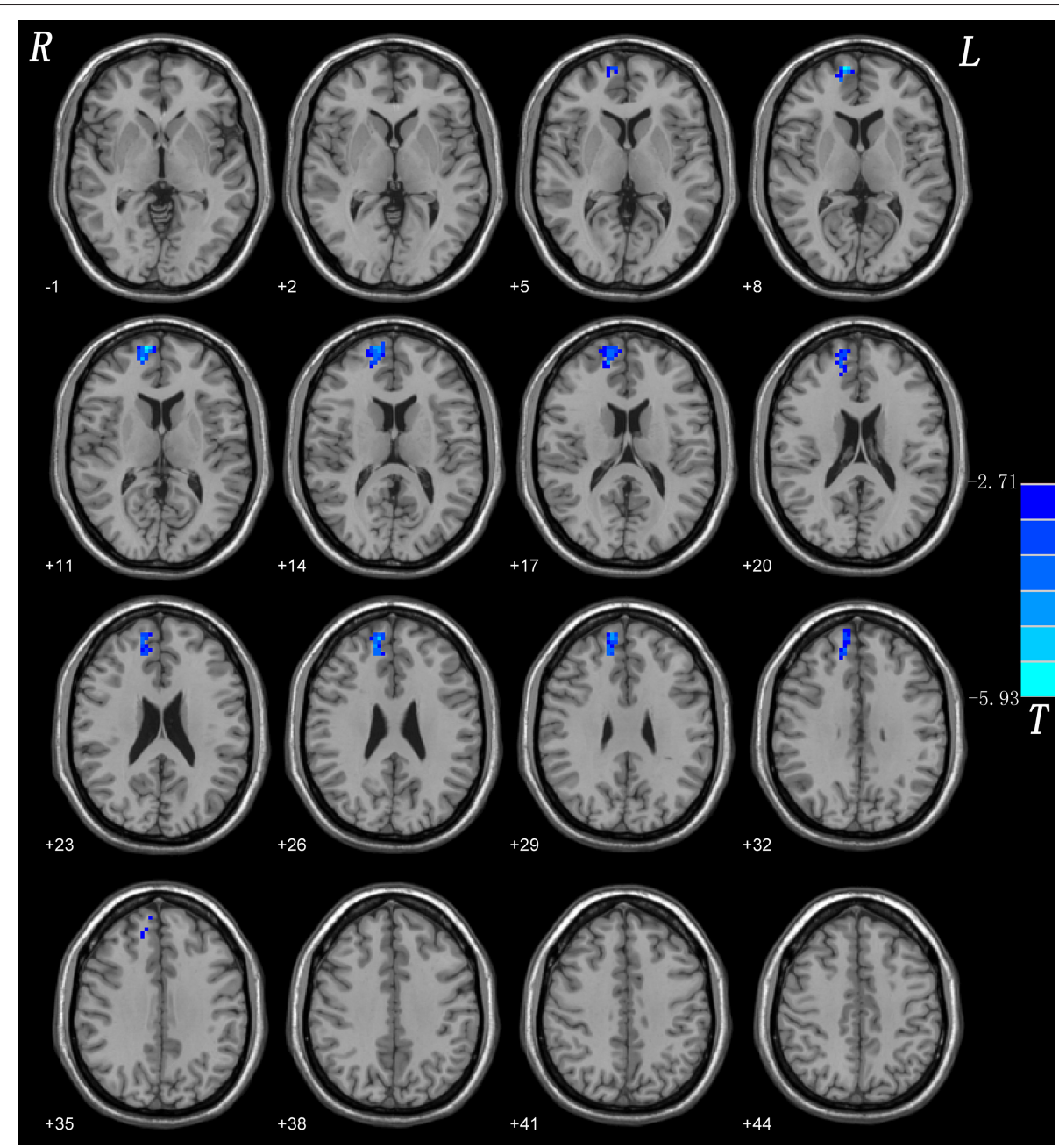

FIGURE 1 | NH differences between patients with CD and controls. Red and blue denote higher and lower $\mathrm{NH}$ respectively and the color bars represent the t values from two-sample $t$-test of the group analysis. $\mathrm{NH}$, network homogeneity; $\mathrm{CD}$, cervical dystonia.

TABLE 3 | Brain regions with significant NH differences in the patients.

Brain regions $\frac{\text { Peak (MNI) Number of voxels T-value }}{x y \quad z}$

Patients $<$ Controls

$\begin{array}{llllll}\text { Right superior medial frontal gyrus } & 12 & 63 & 12 & 90 & -5.9244\end{array}$

$x, y, z$, coordinates of primary peak locations in the MNI space; T, statistical value of peak voxel showing NH differences between the patients with $C D$ and the controls; $C D$, cervical dystonia; NH, network homogeneity; MNI, Montreal neurological Institute.

\section{Reproducibility}

Considering the small sample size of our study, we used splithalf and leave-one-out validations to assess the reproducibility of our results.

\section{Split-Half}

The results of split-half replicated those of the full sample (Figure 2).

\section{Leave-One-Out Validation}

The results indicated a highly reproducible pattern of $\mathrm{NH}$ alteration across these tests, as well as in the overall analysis (Figure 3).

\section{Correlations Between NH and Clinical Variables}

The abnormal NH in the right superior medial frontal gyrus of $\mathrm{CD}$ patients was defined as the ROI, from which mean $\mathrm{NH}$ values were extracted. Linear correlations were calculated between these $\mathrm{NH}$ values and the illness duration or Tsui score in the patient group. No significant correlation was detected between these $\mathrm{NH}$ values and illness duration or Tsui score.

\section{ROC Results}

The abnormality found for $\mathrm{NH}$ values in the right superior medial frontal gyrus of CD patients was further validated by the ROC method. The results supported that the $\mathrm{NH}$ values in the right superior medial frontal gyrus could be applied to differentiate 


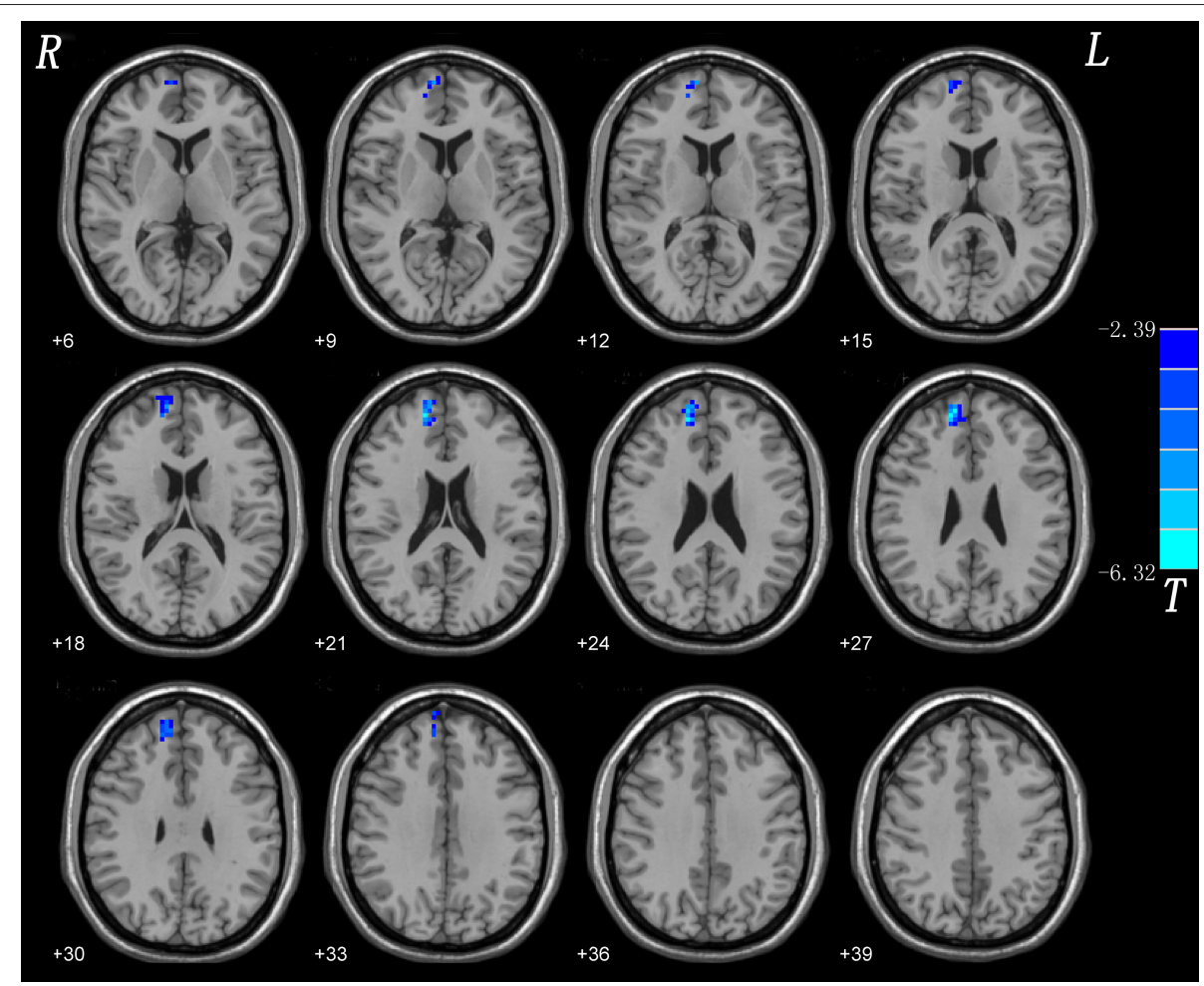

FIGURE 2 | Split-half validation. Comparisons of whole-brain NH were performed between $10 \mathrm{CD}$ patients and 11 healthy controls. Blue denote lower NH respectively and the color bars represent the $\mathrm{t}$ value from two-sample $t$-test of the group analysis. $\mathrm{NH}$, network homogeneity; CD, cervical dystonia.

patients from controls with optimal specificity $(85.71 \%, 18 / 21)$ and sensitivity $(94.74 \%, 18 / 19)$ (Figure 4; Table 4).

\section{DISCUSSION}

Previous fMRI studies in CD patients were mostly task-based, which have limitations compared with resting-state fMRI. First, the introduction of tasks is likely to cause activation in brain regions associated with the completion of tasks and mask abnormal spontaneous activities in regions associated with the pathogenesis of the disease. In addition, it is difficult to choose tasks related to CD symptoms based on MRI scans. Thus, we used the resting-state fMRI method to study $\mathrm{CD}$ patients. $\mathrm{NH}$ analysis is a new method to evaluate the $\mathrm{NH}$ of a distributed and meaningful network based on the level of a voxel-wise comparison. This study used the NH method to analyze the fMRI data of CD patients and HCs. Compared with the HC group, CD patients showed lower $\mathrm{NH}$ values in the right superior medial frontal gyrus. No brain region had higher $\mathrm{NH}$ values in the $\mathrm{CD}$ group compared with those of the HC group. Furthermore, no significant correlations were found between abnormal $\mathrm{NH}$ values and age, illness duration, or symptom severity.

The right superior medial frontal gyrus is a primary region of the SMA $(35,36)$. The SMA, is an important node of the cortical-basal ganglia loop, as well as a complement to the primary motor area and is involved in voluntary movement and advanced motor activities, such as movement and language initiation. This área couples the afferent input and efferent output of the motor cortex area and integrates complicated movement sequences with memory organization (36). Additionally, it is linked to the primary motor cortex, premotor area, and anterior cingulate area. Therefore, decreased $\mathrm{NH}$ values of CD patients in the right superior medial frontal gyrus may be implicated in the involuntary spasms of focal muscles. In the aforementioned studies, the SMA was found to have a decreased function when analyzed using different analytical methods (i.e., VMHC and GFC) (30, 31). These findings strongly suggest that SMA dysfunction plays a key role in CD occurrence.

Of the $19 \mathrm{CD}$ patients we included, 17 patients showed "sensory tricks". Neurophysiological evidence suggests that sensory tricks work by reducing abnormal facilitation. Moreover, this is associated with established dystonia pathogenesis, leading to the hypothesis that sensory tricks reduce abnormally increased facilitation-to-inhibition ratios in brains with dystonia (37). Naumann et al. found that CD patients had a decreased activation of the contralateral SMA, primary sensorimotor cortex, ipsilateral parietal regions while the bilateral occipital lobe was overactivated upon "sensory tricks" stimulation (38). On the basis of these observations, we postulate that $\mathrm{CD}$ patients exhibit disturbed SMA function, leading to abnormal initiation, planning of movement, and advanced motor functions, which may be involved in the mechanism of sensory tricks. Thus, we hypothesize that the right superior medial frontal gyrus affects 


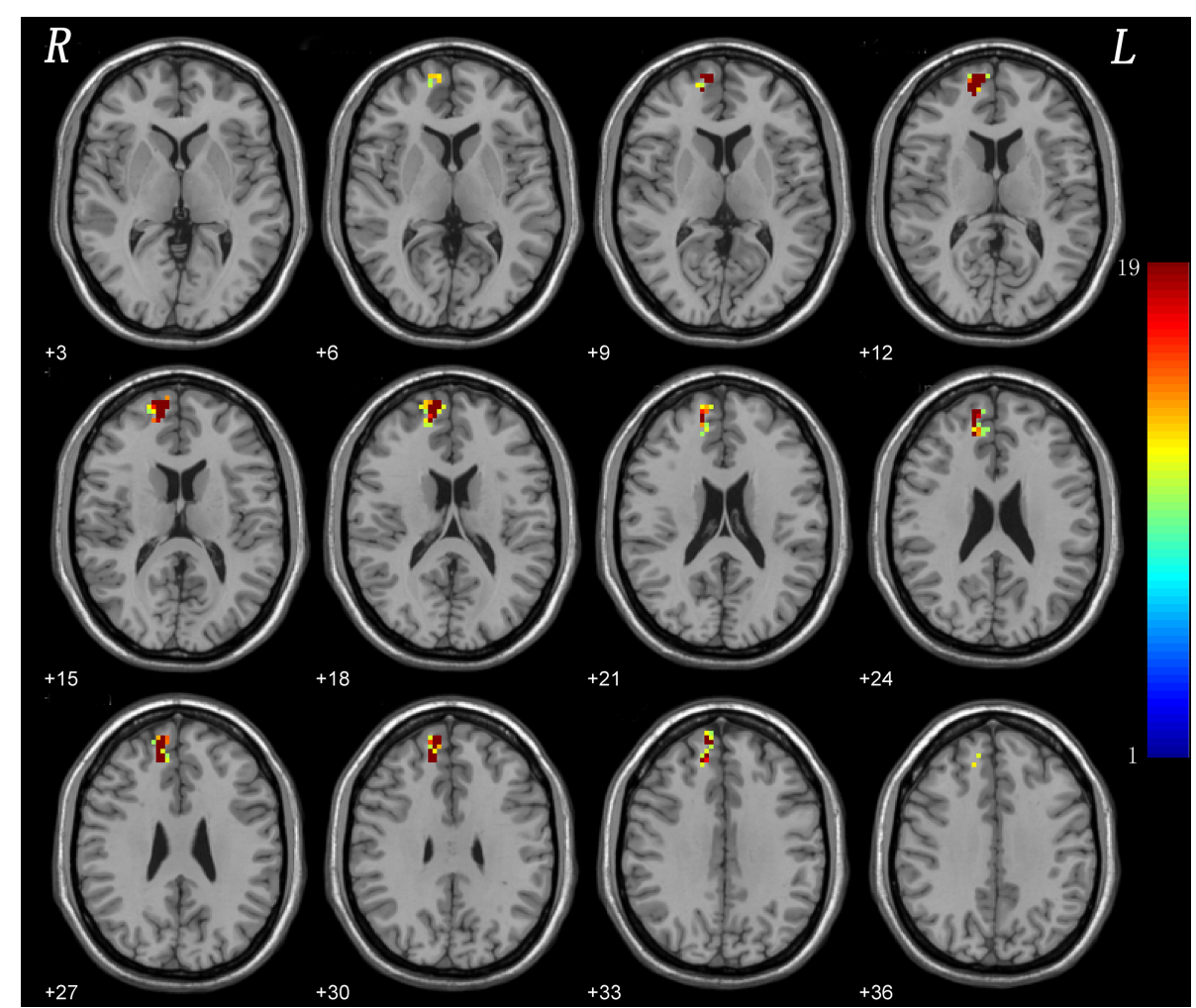

FIGURE 3 | Leave-one-out validation. The group comparisons based upon the permutated samples (i.e., 18 CD vs. 21 healthy controls) for total 19 times. For each voxel, the color indicates number of tests where this voxel exhibited significant group differences across the total 19 tests (i.e., the reproducibility).

the function of the SMA thereby contributing to the onset of CD.

The right superior medial frontal gyrus was suggested as a key node of the DMN (39). Besides, a neuroimaging study that investigated several subregions of the human superior frontal gyrus (SFG) showed a strong functional connectivity with the anterior cingulate cortex of the DMN (40). The DMN performs functional connectivity enhancement and exhibits a high level of neural activity in the resting state but it is inactivated when performing targeted tasks or behaviors. Its main function is to maintain self-referential activity, monitoring the self and surrounding environment (41), and environmental positioning (42). The DMN is known to exhibit abnormal neuro-activity in several neurological and neuropsychiatric disorders, such as Alzheimer's, Parkinson's, epilepsy, schizophrenia (27, 43, 44), and major depressive disorder (28). In another study, we observed abnormal regional activity of this network (the right cerebellum crus I and superior medial prefrontal cortex) (24). Lower $\mathrm{NH}$ values of the right superior medial frontal gyrus in $\mathrm{CD}$ patients might therefore influence the function of this region and result in loss of top-down regulation. This might be a basal pathological change associated with selfreferential activity, self and environmental monitoring, and environmental positioning, consequently disturbing posture control. In another word, lower $\mathrm{NH}$ in the right superior medial frontal gyrus contributed to disturb the DMN function on posture controlling or rather contraction of the cervical musculature involuntary and sustained in CD patients. Thus, the right superior medial frontal gyrus was an evidence of abnormal function of DMN in patients with $\mathrm{CD}$. On this basis, we speculate that the DMN may also be involved in the occurrence of $\mathrm{CD}$ therefore it could be expected as a potential biomarker for further study.

We observed asymmetric activity patterns in $\mathrm{CD}$ that were primarily involved in the right-hemispheric dystoniarelated connectivity pattern. It is important to note that the asymmetric activity patterns were not an accidental finding, as we have noted in other studies in patients with CD (24). This phenomenon has been previously reported, for instance, during finger movements (45) and in the resting state (22). A possible explanation for the laterality of the right hemisphere may be due to its dominance in position control; i.e., the right hemisphere determines response modifications so that it is dominant for position control (46). However, how this pattern relates to the pathogenesis of $\mathrm{CD}$ remains to be further studied.

Strikingly, no significant correlations were found between abnormal NH brain regions and CD clinical data such as illness duration and severity of symptoms. This suggests a corollary that the abnormality of this region is an inherent abnormality of dystonia. The most likely reason is that abnormalities in the cortex and not only the basal ganglia are a cause for 


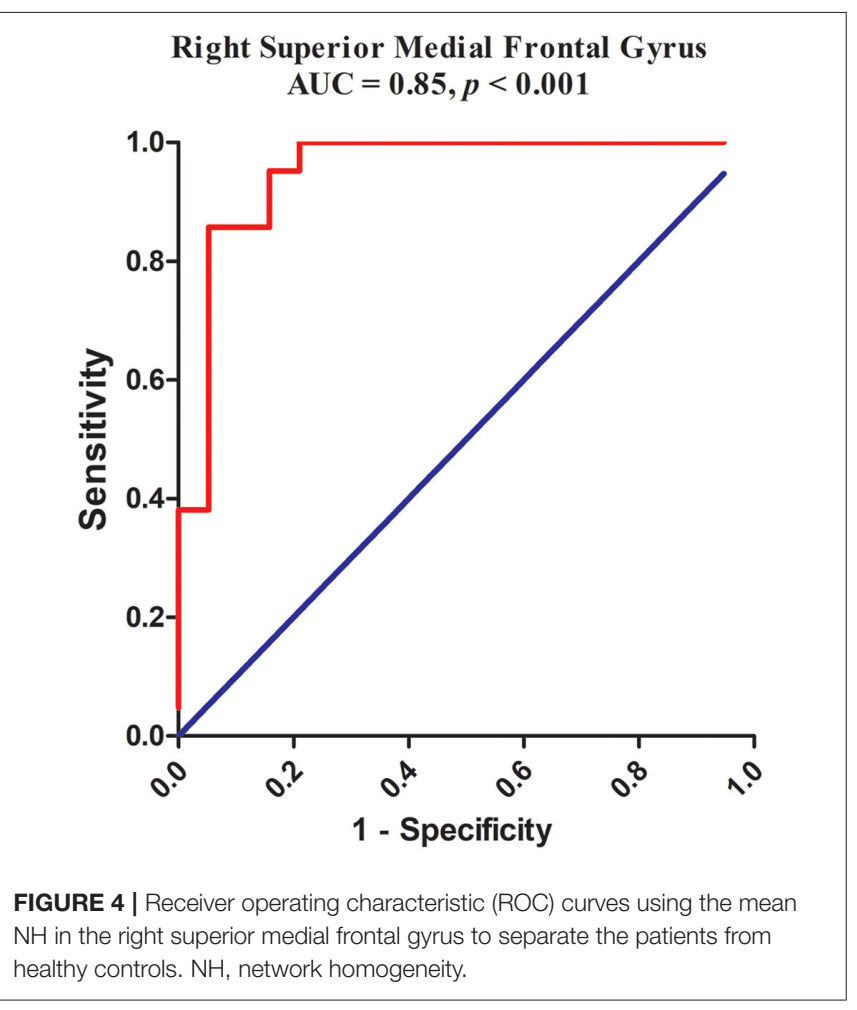

TABLE 4 | ROC analysis for differentiating patients from healthy controls.

\begin{tabular}{lcccc}
\hline Brain regions & $\begin{array}{c}\text { Area under } \\
\text { the curve }\end{array}$ & $\begin{array}{c}\text { Cut-off } \\
\text { point }\end{array}$ & Sensitivity & Specificity \\
\hline $\begin{array}{l}\text { Right medial superior } \\
\text { frontal gyrus }\end{array}$ & 0.950 & 0.80 & $\begin{array}{c}94.74 \%, \\
18 / 19\end{array}$ & $\begin{array}{c}85.71 \%, \\
18 / 21\end{array}$ \\
\hline
\end{tabular}

ROC, receiver operating characteristic; $\mathrm{NH}$, network homogeneity.

this disorder. Thus, we suggest that abnormalities in the right superior medial frontal gyrus are intrinsic to $\mathrm{CD}$. In addition, as presented in Table 3, the sensitivity and specificity of the ROC analysis in the right superior medial frontal gyrus were 94.74 and $85.71 \%$, and the area under the curve of the right superior medial frontal gyrus was $>0.7$, an acceptable accuracy for established diagnostic indicators. Hence, we suggest that decreased $\mathrm{NH}$ in the right superior medial frontal gyrus might be utilized as a potential biomarker to diagnose CD patients.

There are several limitations to our study. First, the sample size was small. Second, dystonic posturing was minimal in the supine position or absent during scanning in all patients. To confirm whether this was a specific sensory trick is challenging. Therefore, the influence of a sensory trick on the analysis could not be fully eliminated. Moreover, physiological noises including

\section{REFERENCES}

1. Chan J, Brin MF, Fahn S. Idiopathic cervical dystonia: clinical characteristics. Move Disord. (1991) 6:119-26. doi: 10.1002/mds.870060206 respiratory and heart rhythms could not be eliminated despite the use of a low sampling rate $(\mathrm{TR}=2 \mathrm{~s})$. Lastly, given the small sample size, patients were not subdivided into different groups according to head rotation. Hence, research using a larger sample size is required to expand our findings.

\section{CONCLUSIONS}

Our findings suggest that abnormal NH of the DMN is a key feature of $C D$ patients, which further highlights the importance of the DMN in the pathology of CD. The DMN may be exploited as a potential biomarker to diagnose $\mathrm{CD}$ patients.

\section{DATA AVAILABILITY STATEMENT}

SL had access to all the data in the study and had final responsibility for decision to submit for publication. The data will be available upon request to SL, luoshuguang@stu.gxmu.edu.cn.

\section{ETHICS STATEMENT}

The studies involving human participants were reviewed and approved by the Ethics Committee of The First Affiliated Hospital, Guangxi Medical University. All participants were given information regarding study procedures and subsequently provided written informed consent to participate in this study.

\section{AUTHOR CONTRIBUTIONS}

SL and WG: conceived and designed the study. SW, XC, YX, WJ, QY, CL, LY, JW, YL, WL, and JT: collected the original imaging data. WG: managed and analyzed the imaging data. SW and XC: wrote the first draft of the manuscript. All authors contributed to the article and approved the submitted version.

\section{FUNDING}

This study was supported by grants from the National Key R\&D Program of China (Grant No. 2016YFC1307100), National Natural Science Foundation of China (Grant No. 81771447), Natural Science Foundation of Hunan (Grant No. 2020JJ4784), Guangxi Appropriate Technology for Medical and Health Research and Development Project (Grant No. S2020028), and Guangxi Appropriate Technology for Medical and Health Research and Development Project (Grant No. S2019002).

\section{ACKNOWLEDGMENTS}

The authors thank all individuals who served as the research participants.

2. Jinnah HA, Berardelli A, Comella C, Defazio G, Delong MR, Factor $\mathrm{S}$, et al. The focal dystonias: current views and challenges for future research. Move Disord. (2013) 28:926-43. doi: 10.1002/mds. 25567 
3. Jankovic J, Leder S, Warner D, Schwartz K. Cervical dystonia: clinical findings and associated movement disorders. Neurology. (1991) 41:1088-91. doi: 10.1212/WNL.41.7.1088

4. Brashear A. Botulinum toxin type $\mathrm{A}$ in the treatment of patients with cervical dystonia. Biologics. (2009) 3:1-7.

5. Charles PD, Adams AM, Davis T, Bradley K, Schwartz M, Brin MF, et al. Neck pain and cervical dystonia: treatment outcomes from cd probe (cervical dystonia patient registry for observation of onabotulinumtoxina efficacy). Pain Pract. (2016) 16:1073-82. doi: 10.1111/papr.12408

6. Martikainen KK, Luukkaala TH, Marttila RJ. Working capacity and cervical dystonia. Parkinsonism Relat Disord. (2010) 16:215-7. doi: 10.1016/j.parkreldis.2009.07.006

7. Richardson SP. Enhanced dorsal premotor-motor inhibition in cervical dystonia. Clin Neurophysiol. (2015) 126:1387-91. doi: 10.1016/ j.clinph.2014.10.140

8. Molho ES, Stacy M, Gillard P, Charles D, Adler CH, Jankovic J, et al. Impact of cervical dystonia on work productivity: an analysis from a patient registry. Mov Disord Clin Pract. (2016) 3:130-8. doi: 10.1002/mdc3. 12238

9. Berardelli A, Rothwell JC, Hallett M, Thompson PD, Manfredi M, Marsden CD. The pathophysiology of primary dystonia. Brain. (1998) 121(Pt 7):1195212. doi: 10.1093/brain/121.7.1195

10. Hallett M. Neurophysiology of dystonia: the role of inhibition. Neurobiol Dis. (2011) 42:177-84. doi: 10.1016/j.nbd.2010.08.025

11. Hendrix CM, Vitek JL. Toward a network model of dystonia. Ann N Y Acad Sci. (2012) 1265:46-55. doi: 10.1111/j.1749-6632.2012. 06692.x

12. Moll CK, Galindo-Leon E, Sharott A, Gulberti A, Buhmann C, Koeppen JA, et al. Asymmetric pallidal neuronal activity in patients with cervical dystonia. Front Syst Neurosci. (2014) 8:15. doi: 10.3389/fnsys.2014. 00015

13. Draganski B, Thun-Hohenstein C, Bogdahn U, Winkler J, May A. "Motor circuit" gray matter changes in idiopathic cervical dystonia. Neurology. (2003) 61:1228-31. doi: 10.1212/01.WNL.0000094240.93745.83

14. Prell T, Peschel T, Kohler B, Bokemeyer MH, Dengler R, Gunther A, et al. Structural brain abnormalities in cervical dystonia. BMC Neurosci. (2013) 14:123. doi: 10.1186/1471-2202-14-123

15. Obermann M, Yaldizli O, de Greiff A, Lachenmayer ML, Buhl AR, Tumczak F, et al. Morphometric changes of sensorimotor structures in focal dystonia. Mov Disord. (2007) 22:1117-23. doi: 10.1002/mds.21495

16. Zoons E, Booij J, Nederveen AJ, Dijk JM, Tijssen MA. Structural, functional and molecular imaging of the brain in primary focal dystoniaa review. Neuroimage. (2011) 56:1011-20. doi: 10.1016/j.neuroimage.2011. 02.045

17. Egger K, Mueller J, Schocke M, Brenneis C, Rinnerthaler M, Seppi K, et al. Voxel based morphometry reveals specific gray matter changes in primary dystonia. Mov Disord. (2007) 22:1538-42. doi: 10.1002/mds.21619

18. Ramdhani RA, Simonyan K. Primary dystonia: conceptualizing the disorder through a structural brain imaging lens. Tremor Other Hyperkinet Mov. (2013) 3:tre-03-152-3638-4. doi: 10.7916/D8H70DJ7

19. Fabbrini G, Pantano P, Totaro P, Calistri V, Colosimo C, Carmellini M, et al. Diffusion tensor imaging in patients with primary cervical dystonia and in patients with blepharospasm. Eur J Neurol. (2008) 15:185-9. doi: 10.1111/j.1468-1331.2007.02034.x

20. Waugh JL, Kuster JK, Levenstein JM, Makris N, Multhaupt-Buell TJ, Sudarsky $\mathrm{LR}$, et al. Thalamic volume is reduced in cervical and laryngeal dystonias. PLoS ONE. (2016) 11:e0155302. doi: 10.1371/journal.pone.0155302

21. Colosimo C, Pantano P, Calistri V, Totaro P, Fabbrini G, Berardelli A. Diffusion tensor imaging in primary cervical dystonia. J Neurol Neurosurg Psychiatry. (2005) 76:1591-3. doi: 10.1136/jnnp.2004.056614

22. Delnooz CC, Pasman JW, Beckmann CF, van de Warrenburg BP. Altered striatal and pallidal connectivity in cervical dystonia. Brain Struct Funct. (2015) 220:513-23. doi: 10.1007/s00429-013-0671-y

23. Delnooz CC, Pasman JW, Beckmann CF, van de Warrenburg BP. Taskfree functional MRI in cervical dystonia reveals multi-network changes that partially normalize with botulinum toxin. PLoS ONE. (2013) 8:e62877. doi: 10.1371/journal.pone.0062877
24. Wei S, Lu C, Chen X, Yang L, Wei J, Jiang W, et al. Abnormal regional homogeneity and its relationship with symptom severity in cervical dystonia: a rest state fMRI study. BMC Neurol. (2021) 21:55. doi: 10.1186/ s12883-021-02079-x

25. Uddin LQ, Kelly AM, Biswal BB, Margulies DS, Shehzad Z, Shaw $D$, et al. Network homogeneity reveals decreased integrity of defaultmode network in ADHD. I Neurosci Methods. (2008) 169:249-54. doi: 10.1016/j.jneumeth.2007.11.031

26. Wei S, Su Q, Jiang M, Liu F, Yao D, Dai Y, et al. Abnormal default-mode network homogeneity and its correlations with personality in drug-naive somatization disorder at rest. J Affect Disord. (2016) 193:81-8. doi: 10.1016/ j.jad.2015.12.052

27. Guo W, Yao D, Jiang J, Su Q, Zhang Z, Zhang J, et al. Abnormal defaultmode network homogeneity in first-episode, drug-naive schizophrenia at rest. Prog Neuropsychopharmacol Biol Psychiatry. (2014) 49:16-20. doi: 10.1016/ j.pnpbp.2013.10.021

28. Guo W, Liu F, Zhang J, Zhang Z, Yu L, Liu J, et al. Abnormal default-mode network homogeneity in first-episode, drug-naive major depressive disorder. PLoS ONE. (2014) 9:e91102. doi: 10.1371/journal.pone.0091102

29. Guo W, Liu F, Yao D, Jiang J, Su Q, Zhang Z, et al. Decreased defaultmode network homogeneity in unaffected siblings of schizophrenia patients at rest. Psychiatry Res. (2014) 224:218-24. doi: 10.1016/j.pscychresns.2014. 08.014

30. Pan P, Wei S, Ou Y, Jiang W, Li W, Lei Y, et al. Reduced global-brain functional connectivity and its relationship with symptomatic severity in cervical dystonia. Front Neurol. (2020) 10:1358. doi: 10.3389/fneur.2019.01358

31. Jiang W, Lei Y, Wei J, Yang L, Wei S, Yin Q, et al. Alterations of interhemispheric functional connectivity and degree centrality in cervical dystonia: a resting-state fMRI study. Neural Plast. (2019) 2019:7349894. doi: 10.1155/2019/7349894

32. Tsui JK, Eisen A, Stoessl AJ, Calne S, Calne DB. Double-blind study of botulinum toxin in spasmodic torticollis. Lancet. (1986) 2:245-7. doi: 10.1016/S0140-6736(86)92070-2

33. Matlab (2012). Available online at: www.mathworks.com

34. Chao-Gan Y, Yu-Feng Z. DPARSF: A MATLAB toolbox for "pipeline" data analysis of resting-state fMRI. Front Syst Neurosci. (2010) 4:13. doi: 10.3389/fnsys.2010.00013

35. Kurata K. Somatotopy in the human supplementary motor area. Trends Neurosci. (1992) 15:159-60. doi: 10.1016/0166-2236(92)90164-4

36. Santosh CG, Rimmington JE, Best JJ. Functional magnetic resonance imaging at $1 \mathrm{~T}$ : motor cortex, supplementary motor area and visual cortex activation. Br J Radiol. (1995) 68:369-74. doi: 10.1259/0007-1285-68808-369

37. Ramos VF, Karp BI, Hallett M. Tricks in dystonia: ordering the complexity. J Neurol Neurosurg Psychiatry. (2014) 85:987-93. doi: 10.1136/ jnnp-2013-306971

38. Naumann M, Magyar-Lehmann S, Reiners K, Erbguth F, Leenders KL. Sensory tricks in cervical dystonia: perceptual dysbalance of parietal cortex modulates frontal motor programming. Ann Neurol. (2000) 47:322-8.

39. Franco AR, Pritchard A, Calhoun VD, Mayer AR. Interrater and intermethod reliability of default mode network selection. Hum Brain Mapp. (2009) 30:2293-303. doi: 10.1002/hbm.20668

40. Li W, Qin W, Liu H, Fan L, Wang J, Jiang T, et al. Subregions of the human superior frontal gyrus and their connections. Neuroimage. (2013) 78:46-58. doi: 10.1016/j.neuroimage.2013.04.011

41. Gusnard DA, Akbudak E, Shulman GL, Raichle ME. Medial prefrontal cortex and self-referential mental activity: relation to a default mode of brain function. Proc Natl Acad Sci U S A. (2001) 98:4259-64. doi: 10.1073/ pnas.071043098

42. Vogt BA, Finch DM, Olson CR. Functional heterogeneity in cingulate cortex: the anterior executive and posterior evaluative regions. Cereb Cortex. (1992) 2:435-43. doi: 10.1093/cercor/2.6.435-a

43. Mohan A, Roberto AJ, Mohan A, Lorenzo A, Jones K, Carney MJ, et al. The significance of the default mode network (DMN) in neurological and neuropsychiatric disorders: a review. Yale J Biol Med. (2016) 89:49-57.

44. Guo W, Xiao C, Liu G, Wooderson SC, Zhang Z, Zhang J, et al. Decreased resting-state interhemispheric coordination in first-episode, drug-naive 
paranoid schizophrenia. Prog Neuropsychopharmacol Biol Psychiatry. (2014) 48:14-9. doi: 10.1016/j.pnpbp.2013.09.012

45. de Vries PM, Johnson KA, de Jong BM, Gieteling EW, Bohning DE, George MS, et al. Changed patterns of cerebral activation related to clinically normal hand movement in cervical dystonia. Clin Neurol Neurosurg. (2008) 110:1208. doi: 10.1016/j.clineuro.2007.09.020

46. Haaland KY, Prestopnik JL, Knight RT, Lee RR. Hemispheric asymmetries for kinematic and positional aspects of reaching. Brain. (2004) 127(Pt 5):1145-58. doi: $10.1093 /$ brain/awh133

Conflict of Interest: The authors declare that the research was conducted in the absence of any commercial or financial relationships that could be construed as a potential conflict of interest.
Publisher's Note: All claims expressed in this article are solely those of the authors and do not necessarily represent those of their affiliated organizations, or those of the publisher, the editors and the reviewers. Any product that may be evaluated in this article, or claim that may be made by its manufacturer, is not guaranteed or endorsed by the publisher.

Copyright $\odot 2021$ Wei, Chen, Xiao, Jiang, Yin, Lu, Yang, Wei, Liu, Li, Tang, Guo and Luo. This is an open-access article distributed under the terms of the Creative Commons Attribution License (CC BY). The use, distribution or reproduction in other forums is permitted, provided the original author(s) and the copyright owner(s) are credited and that the original publication in this journal is cited, in accordance with accepted academic practice. No use, distribution or reproduction is permitted which does not comply with these terms. 